OPEN ACCESS

Edited by:

Priya Ranjit Bhosale,

University of Texas MD Anderson Cancer Center, United States

Reviewed by:

Sanaz Javadi,

University of Texas MD Anderson Cancer Center, United States

Silvana Faria

University of Texas MD Anderson Cancer Center, United States John Lurain

Northwestern Medicine, United States

*Correspondence: Yang Xiang

xiangy@pumch.cn

Wenze Wang

wwzvssxy@126.com

tThese authors share last authorship

Specialty section: This article was submitted to

Women's Cancer,

a section of the journal

Frontiers in Oncology

Received: 25 August 2019 Accepted: 31 October 2019 Published: 15 November 2019

Citation:

Kong Y, Tao G, Zong L, Yang J, Wan X, Wang W and Xiang Y (2019) Diagnosis and Management of Mixed Gestational Trophoblastic Neoplasia: A Study of 16 Cases and a Review of the Literature. Front. Oncol. 9:1262. doi: 10.3389/fonc.2019.01262

\section{Diagnosis and Management of Mixed Gestational Trophoblastic Neoplasia: A Study of 16 Cases and a Review of the Literature}

\author{
Yujia Kong ${ }^{1}$, Guangshi Tao ${ }^{2}$, Liju Zong ${ }^{1}$, Junjun Yang ${ }^{1}$, Xirun Wan ${ }^{1}$, Wenze Wang ${ }^{3 * t}$ and \\ Yang Xiang ${ }^{1 * t}$ \\ ${ }^{1}$ Department of Obstetrics and Gynecology, Peking Union Medical College Hospital, Chinese Academy of Medical Sciences \\ \& Peking Union Medical College, Beijing, China, ${ }^{2}$ Department of Obstetrics and Gynecology, The Second Xiangya Hospital of \\ Central South University, Changsha, China, ${ }^{3}$ Department of Pathology, Peking Union Medical College Hospital, Chinese \\ Academy of Medical Sciences \& Peking Union Medical College, Beijing, China
}

Objective: Mixed gestational trophoblastic neoplasia (GTN) is a rare occurrence that refers to the coexistence of choriocarcinoma and/or placental site trophoblastic tumor and/or epithelioid trophoblastic tumor. The diagnosis and management of mixed GTN are challenging. We investigated the clinicopathological characteristics, diagnoses, treatments, and outcomes of patients with mixed GTN.

Materials and Methods: The medical records and pathological sections of 16 patients with mixed GTN who were treated at Peking Union Medical College Hospital and The Second Xiangya Hospital of Central South University between January 2012 and December 2018 were reviewed.

Results: Pretreatment serum human chorionic gonadotropin (hCG) levels ranged from 180 to $625,024 \mathrm{IU} / \mathrm{L}$, and were $>10,000 \mathrm{IU} / \mathrm{L}$ in 14 of the 16 patients, none of whom were diagnosed correctly at initial presentation. Two patients were diagnosed with choriocarcinoma coexisting with intermediate trophoblastic tumor (ITT) through dilation and curettage (D\&C) before treatment. Another 5 patients were histologically confirmed to have placental site trophoblastic tumor (PSTT) by D\&C but final pathological findings showed mixed PSTT and choriocarcinoma at subsequent hysterectomy. Seven post-chemotherapy patients with an initial clinical diagnosis of choriocarcinoma underwent surgery because of chemoresistance and their pathological findings revealed coexisting ITT. The remaining 2 patients were found to have choriocarcinoma coexisting with ITT following cervical biopsy and pulmonary lobectomy. All patients received chemotherapy: 14 underwent surgery combined with chemotherapy and 2 received chemotherapy alone to preserve fertility. Other than 1 patient who died of disease progression, 15 patients (93.8\%) achieved complete remission (CR) after treatment, although 5 (33.3\%) relapsed. Of these 5 patients with relapse, 3 achieved CR after additional treatment, 1 was receiving an immune checkpoint inhibitor, and 1 was lost to follow-up after refusing further therapy. 
Conclusion: Mixed GTN is difficult to diagnose on initial presentation. Overlap of the ITT component should be considered in refractory chemoresistant choriocarcinoma. Coexistence of choriocarcinoma should be suspected in ITT patients with high hCG levels. Surgery combined with chemotherapy is optimal treatment for choriocarcinoma mixed with ITT.

Keywords: choriocarcinoma, placental site trophoblastic tumor, epithelioid trophoblastic tumor, diagnosis, treatment

\section{INTRODUCTION}

Gestational trophoblastic neoplasia (GTN) refers to a group of uncommon malignant gynecological tumors, including invasive mole, choriocarcinoma, and intermediate trophoblastic tumor (ITT). Specifically, ITT comprise placental site trophoblastic tumor (PSTT) and epithelioid trophoblastic tumor (ETT) (1). Although all choriocarcinoma, PSTT, and ETT arise from abnormal trophoblast proliferation, the diagnosis and management of choriocarcinoma totally differ from those of PSTT or ETT. Choriocarcinoma can be diagnosed based on clinical presentation and markedly elevated serum human chorionic gonadotropin (hCG); therefore, histological evaluation is generally not required for its diagnosis. The first-line treatment for choriocarcinoma is chemotherapy alone, which is sufficient to cure the majority of patients (2). However, PSTT and ETT are rare types of GTN that are diagnosed based on specific pathological evidence. In contrast to patients with choriocarcinoma, serum hCG levels in patients with PSTT and ETT are often normal or only mildly elevated owing to the absence of syncytiotrophoblast (3). Both PSTT and ETT are less sensitive to chemotherapy than choriocarcinoma; hence, hysterectomy is the primary treatment recommended for these tumors without metastases (2-4). Additionally, chemotherapy should be considered for patients with metastases and/or those with high-risk prognostic factors (4-6).

However, some patients may have mixed GTN, which comprise choriocarcinoma and/or PSTT and/or ETT. Such mixed GTNs can be suspected by dilation and curettage (D\&C) before treatment; otherwise, some patients with initially clinical diagnosis of choriocarcinoma undergo postchemotherapy surgery owing to chemoresistance, whereupon postoperative pathological evidence reveals coexisting ITT (thereby explaining the resistance to chemotherapy). However, owing to their rarity, mixed GTNs have mostly been described in case reports to date (7-19), and limited information exists regarding mixed GTN, making their diagnosis and management challenging.

Therefore, we conducted this retrospective study to review a series of patients with mixed GTN treated at 2 Chinese medical centers. To the best of our knowledge, this study contained the largest sample size of patients with mixed GTN investigated to date. We highlight their clinicopathological characteristics, diagnoses, treatments, and outcomes for the first time, and provide further information concerning this rare entity.

\section{MATERIALS AND METHODS}

We conducted a retrospective review of all patients with GTN who were treated at Peking Union Medical College Hospital (PUMCH) Trophoblastic Disease Center and The Second Xiangya Hospital of Central South University from January 2012 to December 2018. Mixed GTNs referred to patients with components of choriocarcinoma and/or PSTT and/or ETT. A total of 16 patients diagnosed with mixed GTN were identified, including 13 from PUMCH and 3 from The Second Xiangya Hospital of Central South University. All 16 patients were confirmed to have choriocarcinoma coexisting with PSTT or ETT upon examination of their pathological sections by gynecologic pathologists. The database and patients' medical records were reviewed to extract the age at diagnosis, antecedent pregnancy, interval between last pregnancy and treatment, presenting symptoms, pre- and post-treatment serum hCG levels, initial diagnosis, pathological diagnosis, International Federation of Gynecology and Obstetrics stage and risk score, treatment, and survival information. All patients provided written consent to using their record data and portions of their surgical specimens for scientific research. This study was performed with the approval of the ethics committees of both participating institutions.

Treatment efficiency was evaluated based on 3 parameters: complete remission (CR), resistance, and relapse. CR referred to normalization of serum hCG for at least 4 consecutive weeks; resistance was considered serum hCG reaching a plateau or increasing after 2-3 courses of chemotherapy; relapse was defined hCG levels rising again after CR.

The patients were followed based on our institutional standards. Serum hCG levels were measured weekly for 1 month, monthly during the following 1 year, followed by every 3 months for the second year, then every 6 months until 5 years and finally once a year afterwards.

\section{RESULTS}

\section{Clinical Characteristics}

The clinical characteristics of the 16 patients with mixed GTN are summarized in Table 1. The mean age at diagnosis was 33.5 years (range, 22-50 years). Abnormal vaginal bleeding was the most common symptom in 14 patients. Moreover, one patient presented with persistent elevated serum hCG after an abortion and another presented with pulmonary nodules. Antecedent pregnancies were completed to term in 7 patients 
TABLE 1 | Characteristics, diagnoses, and treatments of patients with mixed gestational trophoblastic neoplasia in our cohort.

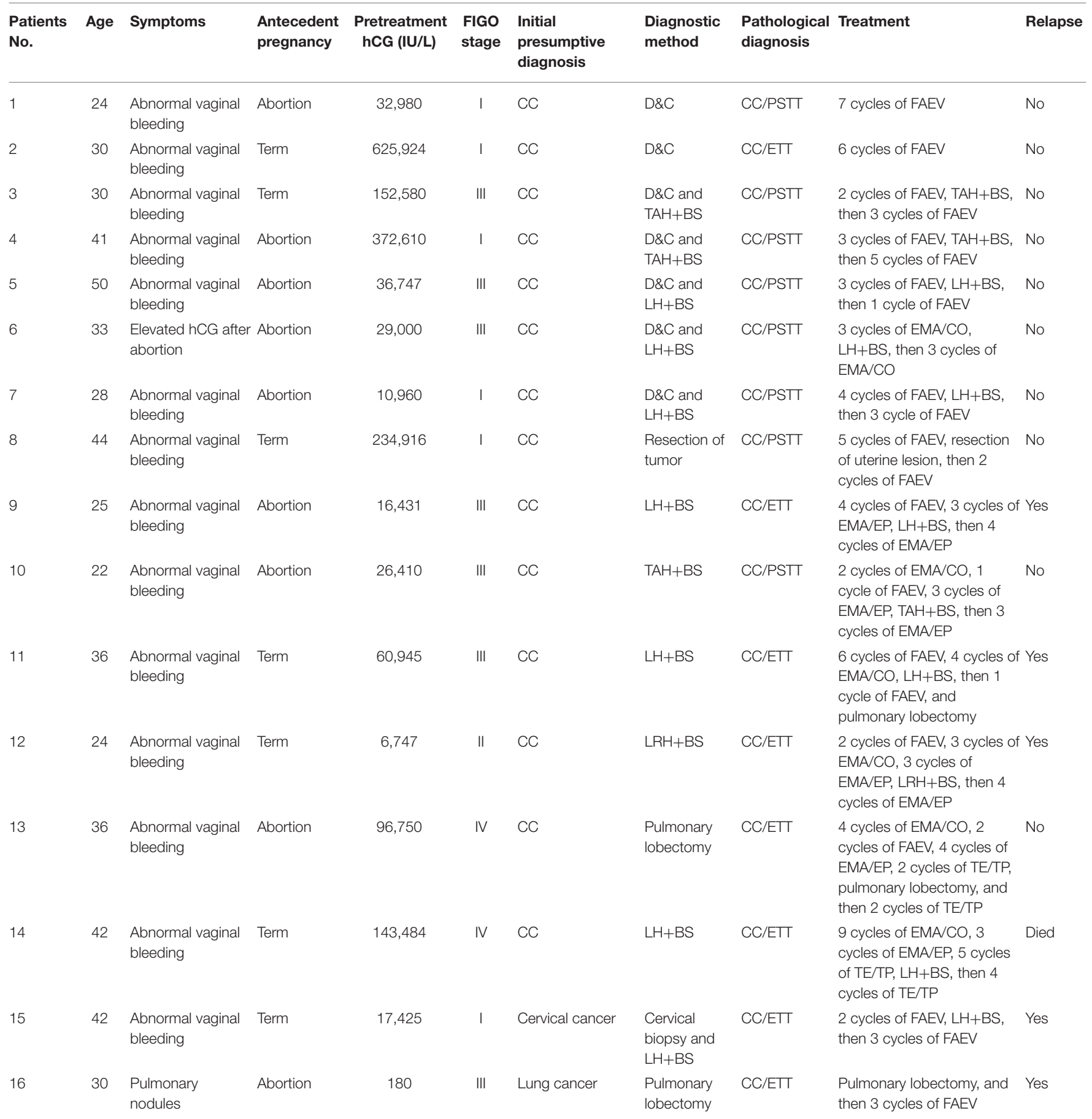

CC, choriocarcinoma; PST, placental site trophoblastic tumor; ETT, epithelioid trophoblastic tumor; D\&C, dilation and curettage; LH, laparoscopic hysterectomy; TAH, total abdominal hysterectomy; $L R H$, laparoscopic radical hysterectomy; BS, bilateral salpingectomy; FAEV, floxuridine, actinomycin-D, etoposide, vincristine; EMA/CO, etoposide, methotrexate, actinomycin-D/cyclophosphamide, vincristine; EMAJEP, etoposide, methotrexate, actinomycin-D/etoposide, cisplatin; TE/TP, paclitaxel, etoposide/paclitaxel, cisplatin.

(43.8\%) and ended in abortion in 9 (56.2\%). None of the patients had an antecedent molar pregnancy. The interval between the antecedent pregnancy and treatment was $\leq 12$ months for 8 patients and $>12$ months in the remaining 8 (50.0\% each). Pretreatment serum hCG levels ranged from 180 to $625,024 \mathrm{IU} / \mathrm{L}$.
Notably, 14 of 16 patients had high levels of hCG $>10,000$ IU/L. The majority of patients presented with uterine lesions, although 2 (patients \#13 and \#16) did not have uterine lesions throughout the course of disease. The size of uterine lesions ranged from 2.5 to $7.0 \mathrm{~cm}$. The tumor was confined to the uterus in 6 patients 
(stage I), 1 patient had vaginal metastases (stage II), 7 patients had lung metastases (stage III), and 2 patients had brain or liver metastases (stage IV).

\section{Diagnostic Evaluation}

The initial diagnoses of the patients with mixed GTN prior to pathological evaluation were suspected choriocarcinoma in 14 , cervical cancer in 1 , and lung cancer in 1 . Among the 16 patients, 2 patients (\#1-2) underwent D\&C owing to irregular vaginal bleeding, whereupon pathological findings revealed choriocarcinoma coexisting with ITT. Another 5 patients (\#37) were histologically confirmed to have PSTT by D\&C. Subsequently, hysterectomy was performed in these patients and the final pathological findings showed PSTT coexisting with choriocarcinoma. Moreover, 7 patients (\#8-14) received chemotherapy as their primary treatment owing to the clinical diagnosis of choriocarcinoma without pathological evidence. However, all of them ultimately underwent hysterectomy or pulmonary lobectomy because of chemoresistance or persistent tumor lesions and the postoperative pathological findings revealed choriocarcinoma coexisting with ITT. One patient (\#15) who presented with a cervical mass underwent a cervical biopsy; her pathological findings was suspected of choriocarcinoma mixed with ETT which was confirmed by pathological samples post-hysterectomy. Another patient (\#16) who was initially suspected of having lung cancer underwent pulmonary lobectomy and was pathologically diagnosed with choriocarcinoma coexisting with ETT.

\section{Pathological Features}

In our retrospective study, choriocarcinoma coexisted with ETT and PSTT in 8 patients each. A variety of histopathological features allowed us to differentiate the components of choriocarcinoma, PSTT, and ETT. Microscopically, the choriocarcinoma component showed a typically biphasic pattern with mononuclear cytotrophoblast and multinuclear syncytiotrophoblast (Figure 1A). Immunohistochemical staining for $\beta$-hCG revealed positive staining in this component (Figure 1B). PSTT was composed of mononuclear trophoblastic cells forming cords and sheets; the tumor cells extensively infiltrated the endometrium and deeply penetrated the uterine wall (Figure 1C). Immunohistochemistry showed positive staining for human placental lactogen (hPL) in intermediate trophoblast of PSTT (Figure 1D). ETT was composed of a relatively uniform population of mononuclear intermediate trophoblastic cells arranged in nests, cords, or masses. These tumor cells contained round nuclei and eosinophilic or clear cytoplasm, and also exhibited high mitotic activity (Figure 1E). Immunohistochemistry showed positive staining for P63 in intermediate trophoblast of ETT (Figure 1F).

\section{Treatment}

All our patients with mixed GTN received chemotherapy; 14 patients underwent surgery combined with chemotherapy while the remaining 2 received only chemotherapy because of their desire to preserve fertility. The most common chemotherapy regimens included: floxuridine, actinomycin-D, etoposide, and vincristine (FAEV); etoposide, methotrexate, actinomycin-D/cyclophosphamide, and vincristine (EMA/CO); and etoposide, methotrexate, actinomycin-D/etoposide, and cisplatin (EMA/EP). Surgeries consisted of simple abdominal or laparoscopic hysterectomies $(n=10)$, laparoscopic radical hysterectomy $(n=1)$, resection of uterine lesion $(n=1)$ and pulmonary lobectomies $(n=3)$. Detailed information regarding the patients' treatments are listed in Table $\mathbf{1 .}$

\section{Outcomes}

All patients were followed via telephone interviews or at clinics. The median follow-up time was 21 months (range, 1-82 months). Fifteen patients (93.8\%) achieved CR after treatment, 5 (33.3\%) of whom relapsed within 3 to 9 months; meanwhile, 1 patient died of disease progression. Among the 5 patients who had relapses, 2 (\#9 and \#12) experienced CR again after chemotherapy and pulmonary lobectomy; 2 (\#11 and \#16) received immune checkpoint inhibitor (pembrolizumab) treatment after relapse and 1 of them (\#11) re-achieved CR; the other 1 (\#15) refused to undergo pulmonary lobectomy and was lost to follow-up. Four of the 5 relapsed patients had extrauterine metastases before treatment and 3 of them were resistant to chemotherapy. None of the 2 patients who underwent fertility-sparing treatments experienced a relapse.

\section{DISCUSSION}

Mixed GTN is a rare occurrence that refers to the coexistence of choriocarcinoma and/or PSTT and/or ETT. To this date, such tumors have mainly been described in individual case reports; hence, information about them is relatively scarce, which complicates their diagnosis and management. For this reason, we conducted this retrospective study and literature review to attain a better understanding of mixed GTNs.

Aside from the 16 patients in our retrospective study, 18 other patients with mixed GTN have previously been reported (7-19). The clinical characteristics, diagnoses, and treatments of patients with mixed GTN reported in the literatures are summarized in Table 2. The majority had a choriocarcinoma component, although 3 patients had ETT and PSTT without choriocarcinoma. The most common symptom was abnormal vaginal bleeding. Sixteen of the 18 patients had elevated serum hCG levels, including 6 with levels $>10,000 \mathrm{IU} / \mathrm{L}$. The interval between the last pregnancy and commencing treatment ranged from 14 weeks to 38 years. The initial diagnoses of these mixed GTNs varied; none were diagnosed correctly at initial presentation. All 3 patients with mixed PSTT and ETT underwent hysterectomy alone, and none relapsed. Among the 15 patients with choriocarcinoma mixed with ITT, 12 received chemotherapy combined with surgery that included hysterectomy $(n=10)$, uterine horn resection $(n=1)$, and pulmonary lobectomy $(n=1) ; 2$ patients underwent only hysterectomy without chemotherapy, and 1 with extensive metastases received only chemotherapy. Among the 15 patients whose survival information was available, 14 patients achieved $\mathrm{CR}$ and 1 patient died of disease progression after treatment. After a median follow-up of 45 months (range, 1-238 months) 
A

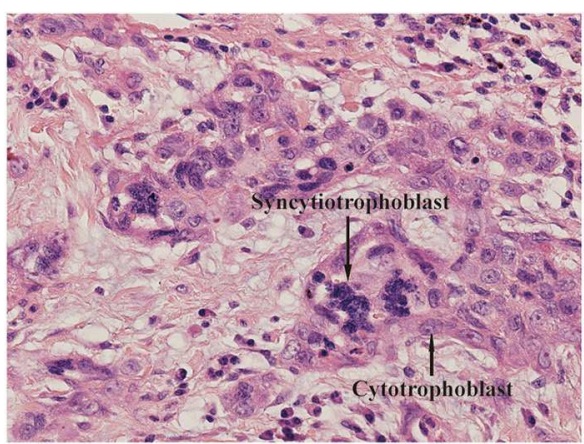

C

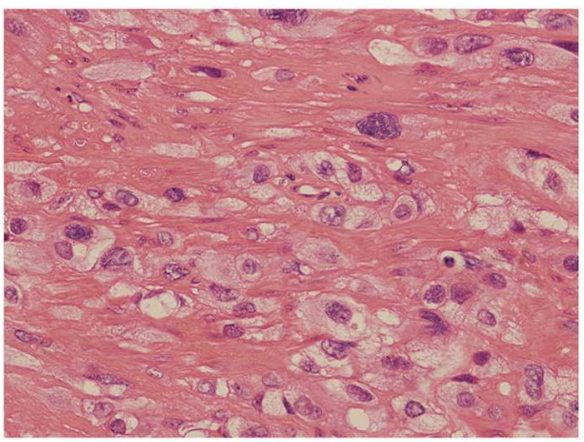

E

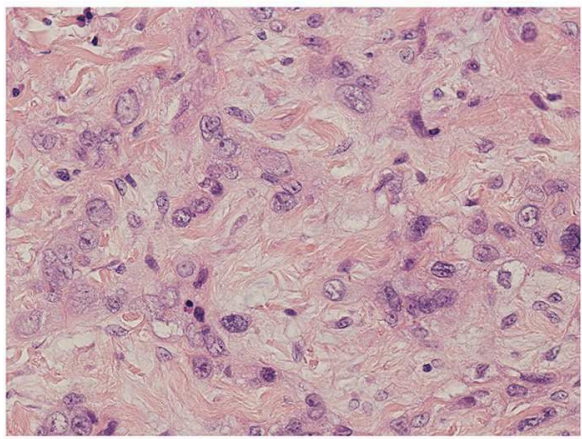

B

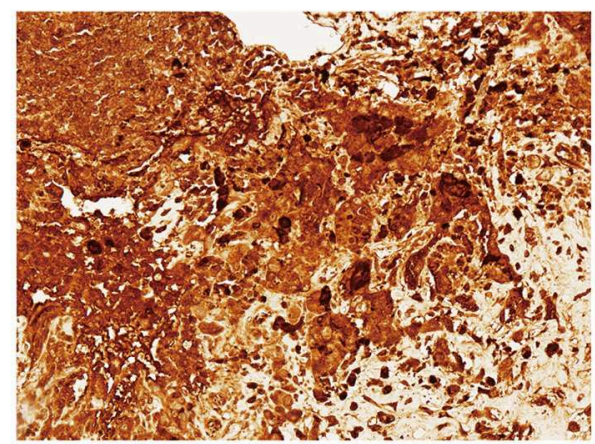

D

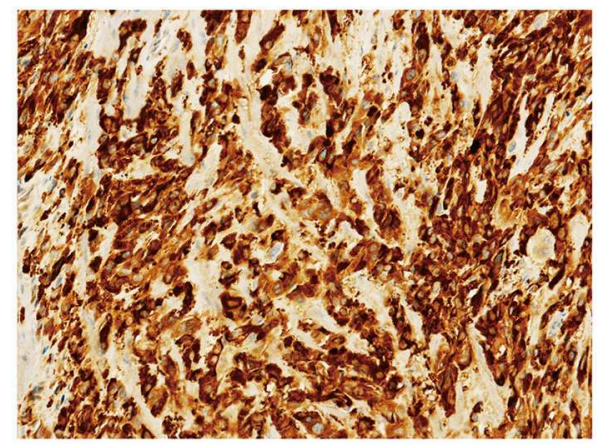

$\mathbf{F}$

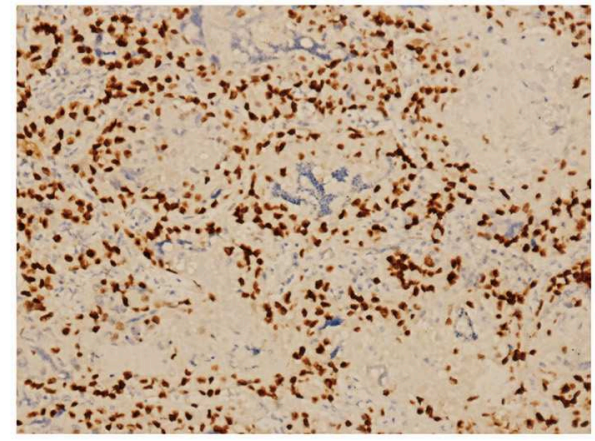

FIGURE 1 | (A) Choriocarcinoma component showed biphasic pattern of mononuclear cytotrophoblast and multinuclear syncytiotrophoblast [hematoxylin-eosin (H\&E), original magnification $\times 300$ ]. (B) Immunohistochemistry showed positive staining for $\beta$-hCG in choriocarcinoma (original magnification $\times 150)$. (C) PSTT component showed cords and sheets of monomorphic intermediate trophoblast with pleomorphic nuclei. The tumor cells dissected and separated the smooth muscle bundles. (H\&E, original magnification $\times 300$ ). (D) Immunohistochemistry showed positive staining for hPL in intermediate trophoblast of PSTT (original magnification $\times 150)$. (E) ETT component showed nests and cords of uniform mononucleate intermediate trophoblastic cells with intercellular eosinophilic hyaline or fibrillar material (H\&E, original magnification $\times 300)$. (F) Immunohistochemistry showed positive staining for P63 in intermediate trophoblast of ETT (original magnification ×150). PST, placental site trophoblastic tumor; ETT, epithelioid trophoblastic tumor; hCG, human chorionic gonadotropin; hPL, human placental lactogen.

for the 14 patients, 10 were alive without disease; 4 relapsed after remission and yet were alive after salvage treatment.

PSTT and ETT are derived from intermediate trophoblast, therefore, hCG levels in patients with these 2 rare types of GTN are often normal or only mildly elevated. Horowitz et al. reported that hCG levels were $<1,000 \mathrm{IU} / \mathrm{L}$ in most patients with PSTT and ETT, while high hCG levels (>10,000 IU/L) were often found in patients with choriocarcinoma $(3,20)$. Among previously reported patients with mixed GTN as well as those in our series, the majority had markedly elevated
hCG levels (>10,000 IU/L); such patients are more likely to be suspected of having choriocarcinoma on initial clinical presentation before pathological evidence becomes available. However, owing to the coexistence of ITT with choriocarcinoma, they are unlikely to be as sensitive to chemotherapy. In our cohort, 7 patients with a clinical diagnosis of choriocarcinoma were ultimately found to be chemoresistant, following which coexisting ITT was found with choriocarcinoma on pathological samples post-surgery. Based on these results, we conclude that histologic evaluation is necessary for choriocarcinomas 
TABLE 2 | Characteristics, diagnoses, and treatments of patients with mixed gestational trophoblastic neoplasia reported in the literature.

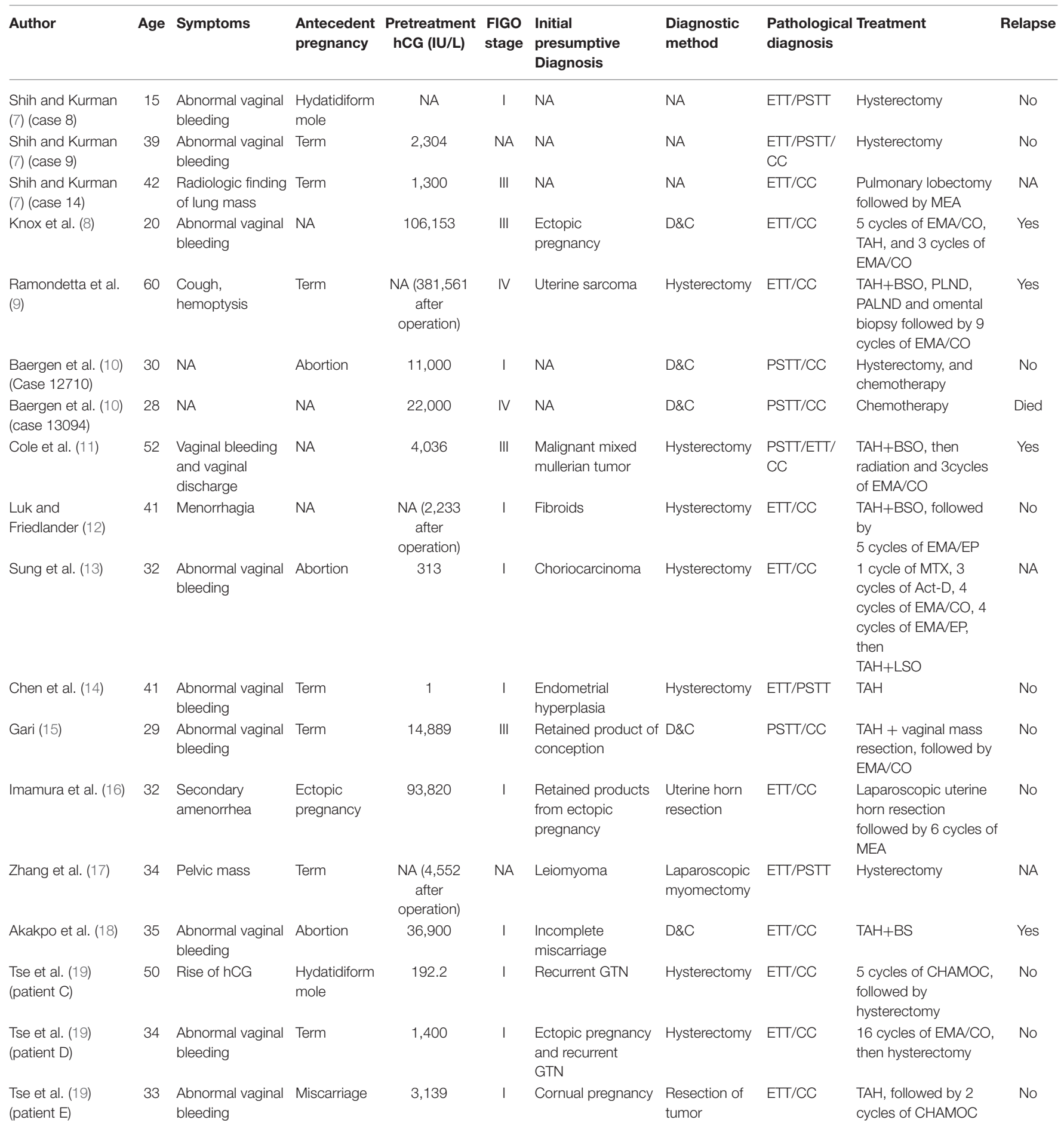

CC, choriocarcinoma; PST, placental site trophoblastic tumor; ETT, epithelioid trophoblastic tumor; D\&C, dilation and curettage; Act-D, actinomycin-D; MTX, methotrexate; MEA, methotrexate, etoposide and actinomycin-D; EMA/CO, etoposide, methotrexate, actinomycin-D/cyclophosphamide, vincristine; EMA/EP, etoposide, methotrexate, actinomycinD/etoposide, cisplatin; CHAMOC, cyclophosphamide, hydroxyurea, actinomycin-D, methotrexate with folinic acid, and vincristine; TAH, total abdominal hysterectomy; BS, bilateral salpingectomy; BSO, bilateral salpingo-oophorectomy; LSO, left salpingo-oophorectomy; PLND, pelvic lymph node dissection; PALND, para-aortic lymph node dissection; NA, not available.

that are resistant to chemotherapy. Pathologists specializing in gynecological oncology should review these specimens carefully to avoid missing any coexisting ITT component that may be present. Furthermore, among choriocarcinomas mixed with ITT, sensitivity to chemotherapy might be associated with the ratio of syncytiotrophoblast/cytotrophoblast-to-intermediate 
trophoblast (9). Therefore, it is recommended that pathologists investigate the proportions of different mixed GTN components in order to determine whether the clinical behaviors of these tumors are governed by the dominant component.

Given the chemoresistant nature of PSTT and ETT, surgery is the primary treatment modality. Previous studies demonstrated that patients with uterine-confined PSTT or ETT can be cured by primary hysterectomy alone without chemotherapy $(3,5,6,21)$. In our series, the majority of patients with mixed GTN underwent surgeries that included hysterectomy and pulmonary lobectomy. Only 2 patients received chemotherapy alone to preserve fertility, none of whom experienced relapse. The favorable responses to chemotherapy in these 2 patients may be related to the fact that choriocarcinoma was the predominant component. Moreover, chemotherapy should be combined with surgery for patients with choriocarcinoma coexisting with ITT, although adjuvant chemotherapy appears to provide no benefit for patients with uterine-confined tumors composed purely of PSTT or ETT (3). Most patients with mixed GTN usually have high hCG levels owing to the choriocarcinoma component. Feng and Xiang suggested that hCG should be maintained at low levels before surgery to achieve good outcomes (22). Therefore, 13 of the 14 patients in our cohort who underwent surgery received chemotherapy beforehand to lower preoperative hCG levels to the greatest extent possible. Hence, we recommend that surgery for patients with choriocarcinoma coexisting with ITT should be performed after preoperative hCG levels have been maintained at their lowest possible values post-chemotherapy.

In terms of prognosis, $33.3 \%$ of patients with mixed GTN in our cohort experienced relapse; this rate appeared to be much higher than that of patients with pure choriocarcinoma or ITT. However, it is difficult to draw the conclusion that the prognoses of patients with mixed GTN are necessarily poor owing to the small sample size in our study. Therefore, more international cooperation that can yield larger studies is required to attain a better understanding of this entity.

Almost all reported mixed GTNs comprised choriocarcinoma and ITT; the exceptions were 3 previously reported patients who had only PSTT and ETT $(7,14,17)$. The pathogenesis of mixed GTN is poorly understood. Mao et al. proposed that choriocarcinoma is the most primitive trophoblastic tumor, and is composed of varied amounts of cytotrophoblast, syncytiotrophoblast, and intermediate trophoblast. In contrast, PSTT and ETT are relatively more differentiated. PSTT involves a neoplastic cytotrophoblast differentiating into intermediate trophoblastic cells at the site of implantation, while ETT involves the neoplastic cytotrophoblast differentiating into chorionictype intermediate trophoblastic cells $(23,24)$. The model may explain coexistence of mixed GTN. Furthermore, in our series, some post-chemotherapy choriocarcinomas were found to be coexisting with ITT postoperatively. Based on Shih and Kurman's hypothesis (25), it is speculated that multiple courses of chemotherapy may have destroyed most sensitive choriocarcinoma tumor cells; the remaining cells differentiated into intermediate trophoblastic cells that were refractory to chemotherapy. Lu et al. also reported that 4 chemoresistant GTN patients were found to have coexisting ETT postoperatively (26), which further supports the notion that ETT developed from a preexisting choriocarcinoma after chemotherapy.

Our study had certain limitations. Given the rarity of GTNs, we were only able to conduct a retrospective study. Furthermore, it was difficult to draw conclusions regarding the prognoses of patients with mixed GTN owing to the small sample size. However, to the best of our knowledge, our study comprised the largest sample size investigated to date, and we also reviewed all previously reported patients with mixed GTN; hence, our study ought to provide valuable information toward understanding this entity.

In conclusion, the coexistence of choriocarcinoma and/or PSTT and/or ETT is rare, and such patients are difficult to diagnose correctly at initial presentation. On one hand, the presence of coexisting ITT component should be considered in patients with refractory chemoresistant choriocarcinoma, and histological evaluation should be performed. On the other hand, coexisting choriocarcinoma should be suspected in ITT patients with high hCG levels; pathologists should review the specimens carefully so as not to miss the possibility of coexisting choriocarcinoma. Surgery combined with chemotherapy is the best treatment regimen for choriocarcinoma coexisting with ITT.

\section{DATA AVAILABILITY STATEMENT}

The raw data supporting the conclusions of this manuscript will be made available by the authors, without undue reservation, to any qualified researcher.

\section{ETHICS STATEMENT}

The studies involving human participants were reviewed and approved by The Ethics Committees of Peking Union Medical College Hospital and The Ethics Committees of The Second Xiangya Hospital of Central South University. The patients/participants provided their written informed consent to participate in this study. Written informed consent was obtained from the individual(s) for the publication of any potentially identifiable images or data included in this article.

\section{AUTHOR CONTRIBUTIONS}

YK and YX contributed to the conception and design of this study. YK, GT, JY, and XW contributed to data acquisition. YK and WW contributed to data interpretation and analysis. WW and YX contributed to study supervision. YK and LZ contributed to manuscript editing. All authors contributed to manuscript review.

\section{FUNDING}

This work was supported by grants from the National Natural Science Foundation of China (No. 81672586) and the Chinese Academy of Medical Sciences Initiative for Innovative Medicine (CAMS-2017-I2M-1-002) to YX. 


\section{REFERENCES}

1. Osborne R, Dodge J. Gestational trophoblastic neoplasia. Obstet Gynecol Clin North Am. (2012) 39:195-212. doi: 10.1016/j.ogc.2012.03.002

2. Ngan HYS, Seckl MJ, Berkowitz RS, Xiang Y, Golfier F, Sekharan PK, et al. Update on the diagnosis and management of gestational trophoblastic disease. Int J Gynaecol Obstet. (2018) 143(Suppl. 2):79-85. doi: 10.1002/ijgo. 12615

3. Horowitz NS, Goldstein DP, Berkowitz RS. Placental site trophoblastic tumors and epithelioid trophoblastic tumors: biology, natural history, and treatment modalities. Gynecol Oncol. (2017) 144:208-14. doi: 10.1016/j.ygyno.2016.10.024

4. Lurain JR. Gestational trophoblastic disease II: classification and management of gestational trophoblastic neoplasia. Am J Obstet Gynecol. (2011) 204:11-8. doi: 10.1016/j.ajog.2010.06.072

5. Zhao J, Lv WG, Feng FZ, Wan XR, Liu JH, Yi XF, et al. Placental site trophoblastic tumor: a review of 108 cases and their implications for prognosis and treatment. Gynecol Oncol. (2016) 142:102-8. doi: 10.1016/j.ygyno.2016.05.006

6. Yang J, Zong L, Wang J, Wan X, Feng F, Xiang Y. Epithelioid trophoblastic tumors: treatments, outcomes, and potential therapeutic targets. J Cancer. (2019) 10:11-9. doi: 10.7150/jca.28134

7. Shih IM, Kurman RJ. Epithelioid trophoblastic tumor: a neoplasm distinct from choriocarcinoma and placental site trophoblastic tumor simulating carcinoma. Am J Surg Pathol. (1998) 22:1393-403. doi: 10.1097/00000478-199811000-00010

8. Knox S, Brooks SE, Wong-You-Cheong J, Ioffe O, Meisenberg B, Goldstein DP. Choriocarcinoma and epithelial trophoblastic tumor: successful treatment of relapse with hysterectomy and high-dose chemotherapy with peripheral stem cell support: a case report. Gynecol Oncol. (2002) 85:204-8. doi: 10.1006/gyno.2002.6583

9. Ramondetta LM, Silva EG, Levenback CF, Burke TW. Mixed choriocarcinoma in a postmenopausal patient. Int J Gynecol Cancer. (2002) 12:312-6. doi: 10.1046/j.1525-1438.2002.01110.x

10. Baergen RN, Rutgers JL, Young RH, Osann K, Scully RE. Placental site trophoblastic tumor: a study of 55 cases and review of the literature emphasizing factors of prognostic significance. Gynecol Oncol. (2006) 100:511-20. doi: 10.1016/j.ygyno.2005.08.058

11. Cole ME, Broaddus R, Thaker P, Landen C, Freedman RS. Placental-site trophoblastic tumors: a case of resistant pulmonary metastasis. Nat Clin Pract Oncol. (2008) 5:171-5. doi: 10.1038/ncponc1042

12. Luk WY, Friedlander M. A fibroid or cancer? A rare case of mixed choriocarcinoma and epithelioid trophoblastic tumour. Case Rep Obstet Gynecol. (2013) 2013:492754. doi: 10.1155/2013/492754

13. Sung WJ, Shin HC, Kim M-K, Kim MJ. Epithelioid trophoblastic tumor: clinicopathologic and immunohistochemical analysis of three cases. Kor $J$ Pathol. (2013) 47:67-73. doi: 10.4132/KoreanJPathol.2013.47.1.67

14. Chen BJ, Cheng CJ, Chen WY. Transformation of a post-cesarean section placental site nodule into a coexisting epithelioid trophoblastic tumor and placental site trophoblastic tumor: a case report. Diagn Pathol. (2013) 8:85. doi: 10.1186/1746-1596-8-85

15. Gari A. Placental site trophoblastic tumor and choriocarcinoma: an unusual presentation. BMC Res Notes. (2015) 8:703. doi: 10.1186/s13104-015-1693-8
16. Imamura $\mathrm{Y}$, Tashiro $\mathrm{H}$, Saito $\mathrm{F}$, Takaishi $\mathrm{K}$, Ohba $\mathrm{T}$, Fukunaga $\mathrm{M}$, et al. Choriocarcinoma coexisting with epithelioid trophoblastic tumor of the uterine horn. Gynecol Oncol Rep. (2015) 14:31-3. doi: 10.1016/j.gore.2015.10.002

17. Zhang X, Zhou C, Yu M, Chen X. Coexisting epithelioid trophoblastic tumor and placental site trophoblastic tumor of the uterus following a term pregnancy: report of a case and review of literature. Int J Clin Exp Pathol. (2015) 8:7254-9.

18. Akakpo PK, Ulzen-Appiah K, Agbeno E, Derkyi-Kwarteng L. Diagnosing and treating rare lesions in a low resource setting: lessons from a hybrid epithelioid trophoblastic tumor and choriocarcinoma. Ghana Med J. (2017) 51:196-9.

19. Tse KY, Chiu KWH, Chan KKL, Chu MMY, Ngu SF, Cheung ANY, et al. A case series of five patients with pure or mixed gestational epithelioid trophoblastic tumors and a literature review on mixed tumors. Am J Clin Pathol. (2018) 150:318-32. doi: 10.1093/ajcp/aqy039

20. Frijstein MM, Lok CAR, Trommel NE, Kate-Booij MJ, Massuger LFAG Werkhoven E, et al. Management and prognostic factors of epithelioid trophoblastic tumors: results from the International Society for the Study of Trophoblastic Diseases database. Gynecol Oncol. (2018) 152:361-7. doi: 10.1016/j.ygyno.2018.11.015

21. Davis MR, Howitt BE, Quade BJ, Crum CP, Horowitz NS, Goldstein DP, et al. Epithelioid trophoblastic tumor: a single institution case series at the New England Trophoblastic Disease Center. Gynecol Oncol. (2015) 137:456-61. doi: 10.1016/j.ygyno.2015.03.006

22. Feng F, Xiang Y. Surgical management of chemotherapy-resistant gestational trophoblastic neoplasia. Expert Rev Anticancer Ther. (2010) 10:71-80. doi: 10.1586/era.09.169

23. Mao TL, Kurman RJ, Huang CC, Lin MC, Shih Ie M. Immunohistochemistry of choriocarcinoma: an aid in differential diagnosis and in elucidating pathogenesis. Am J Surg Pathol. (2007) 31:1726-32. doi: 10.1097/PAS.0b013e318058a529

24. Shih I-M. Gestational trophoblastic neoplasia-pathogenesis and potential therapeutic targets. Lancet Oncol. (2007) 8:642-50. doi: 10.1016/S1470-2045(07)70204-8

25. Shih IM, Kurman RJ. The pathology of intermediate trophoblastic tumors and tumor-like lesions. Int J Gynecol Pathol. (2001) 20:31-47. doi: 10.1097/00004347-200101000-00004

26. Lu B, Zhang X, Liang Y. Clinicopathologic analysis of postchemotherapy gestational trophoblastic neoplasia: an entity overlapping with epithelioid trophoblastic tumor. Int J Gynecol Pathol. (2016) 35:516-24. doi: 10.1097/PGP.0000000000000259

Conflict of Interest: The authors declare that the research was conducted in the absence of any commercial or financial relationships that could be construed as a potential conflict of interest.

Copyright (c) 2019 Kong, Tao, Zong, Yang, Wan, Wang and Xiang. This is an openaccess article distributed under the terms of the Creative Commons Attribution License (CC BY). The use, distribution or reproduction in other forums is permitted, provided the original author(s) and the copyright owner(s) are credited and that the original publication in this journal is cited, in accordance with accepted academic practice. No use, distribution or reproduction is permitted which does not comply with these terms. 